\title{
PCSK9-Hemmer erstmals in Lipidleitlinie
}

\author{
In der aktuellen europäischen Lipidleitlinie werden erstmals PCSK9-Hem- \\ mer empfohlen. Als Option gelten die neuen Medikamente bei hohen LDL- \\ Werten trotz Statintherapie oder aufgrund einer familiären Belastung.
}

verordnen, sofern andere Therapien und eine günstige Ernährungsweise das LDLCholesterin nicht ausreichend senken.

\section{Empfohlen bei sehr hohem Risiko}

Etwas klarer gibt sich die aktuelle Leitlinie der Europäischen Gesellschaften für Kardiologie (ESC) und Atherosklerose (EAS). Sie hat erstmals die Therapie mit den neuen Arzneien berücksichtigt. Bei Patienten mit sehr hohem kardiovaskulärem Risiko kann danach eine Behandlung mit PCSK9-Blockern erwogen werden, wenn trotz einer Statin-Maximaldosis oder einer Kombination von hoch dosiertem Statin und Ezetimib hohe LDL-Werte persistieren.

Bestehen hohe LDL-Konzentrationen aufgrund einer familiären Hypercholes-

\section{Große LDL-Schwankungen - schlechtes Gedächtnis}

Ältere Patienten mit starken LDL-Schwankungen haben möglicherweise ein erhöhtes Demenzrisiko: Ihre kognitive Leistung ist verringert, der zerebrale Blutfluss vermindert und das Hirn zeigt verstärkt Leukoaraiosen.

Forscher um Dr. Roelof Smit von der Universität in Leiden haben die LDL-Variabilität mit Blick auf die kognitive Leistung bei älteren Personen (70-80Jahre) untersucht. Als Grundlage wählten sie Daten der PROSPER-Studie, in der Pravastatin gegen Placebo geprüft worden war. Alle Teilnehmer wiesen mehrere kardiovaskuläre Risikofaktoren wie Hypertonie, Diabetes oder Rauchen auf. Durch regelmäßige LDL-Kontrollen und kognitive Tests zum Studienbeginn sowie nach 3, 6, 12 und 24 Monaten wurden die LDL-Schwankungen mit den kognitiven Leistungen in Beziehung gesetzt.

Es zeigte sich, dass Patienten in dem Terzil mit der höchsten LDL-Variabilität auch unter Berücksichtigung weiterer Risikofaktoren deutliche kognitive Defizite aufwiesen: Sie schnitten signifikant schlechter in Gedächtnis- und Aufmerksamkeitstests ab. In einer nach drei Jahren durchgeführten MRT-Untersuchung war bei den Patienten mit den höchsten LDL-Schwankungen zudem der zerebrale Blutfluss am geringsten und das Ausmaß der Hyperintensitäten in der weißen Substanz am höchsten.

mut

- Circulation 2016;134:212-221

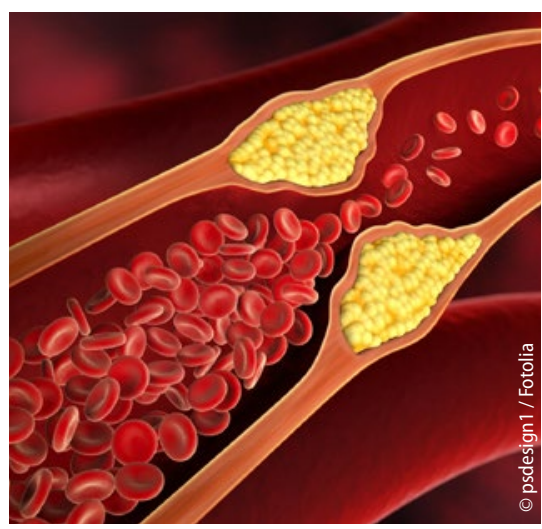

Mit dem LDL sinkt das Gefäßrisiko.

terinämie, legen die Leitlinienautoren ebenfalls eine Therapie mit den Antikörpern nahe, und zwar dann, wenn ein sehr hohes kardiovaskuläres Risiko besteht, eine KHK in der Familie schon in jungem Alter beobachtet wird, die LDLWerte trotz konventioneller Behandlung weit jenseits der Zielwerte liegen, Statine nicht vertragen werden oder hohe Lipoprotein-A-Spiegel gemessen werden.

Die Therapie mit PCSK9-Hemmern wird in der Regel in Kombination mit anderen Lipidsenkern empfohlen, sofern diese vertragen werden und nicht kontraindiziert sind.

\section{Geänderte LDL-Ziele}

Relevant für die Therapie mit den neuen Arzneien dürften auch Änderungen bei den LDL-Zielen sein. Galt bisher bei sehr hohem kardiovaskulärem Risiko ein Zielwert von weniger als $70 \mathrm{mg} / \mathrm{dl}$ oder zumindest eine 50\%-Reduktion, sofern sich der Zielwert nicht erreichen lässt, so sollten Ärzte nun auch dann eine 50\%-Reduktion anstreben, wenn der LDL-Wert zu Beginn zwischen 70 und $135 \mathrm{mg} / \mathrm{dl}$ liegt. In anderen Worten: Bei allen Hochrisikopatienten mit relativ niedrigen LDL-Ausgangswerten macht eine Halbierung der LDL-Serumwerte mehr Sinn als ein fixes Ziel von weniger als $70 \mathrm{mg} / \mathrm{dl}$. Individuell können die Zielwerte also deutlich niedriger liegen. Bei Basis-LDL-Werten über $135 \mathrm{mg} / \mathrm{dl}$ gilt weiterhin ein Ziel von unter $70 \mathrm{mg} / \mathrm{dl}$.

Thomas Müller

- Catapano AL et al. 2016 ESC/EAS Guidelines for the Management of Dyslipidaemias. Eur Heart J 2016;37(39):2999-3058.

https://doi.org/10.1093/eurheartj/ehw272 